\title{
POSITRON ANNIHILATION IN OBSIDIANS
}

\author{
J. CHOJCAN \\ Institute of Experimental Physics, University of Wrocław \\ Pl. Maxa Borna 9, 50-204 Wroclaw, Poland \\ AND M. SACHANBIŃSKI \\ Institute of Geological Sciences, University of Wrocław \\ Pl. Maxa Borna 9, 50-204 Wrocław, Poland
}

\begin{abstract}
Positron lifetime spectra as well as one-dimensional angular correlations of positron annihilation radiation were measured at room temperature for samples of rhyolitic obsidian coming from Iceland, Italy and Armenia. The samples were of different age. One specimen (originating from Iceland) was relatively young, formed in $\mathbf{1 9 8 0}$. The others were much older, collected during the 19th century. It was found that the linear dimensions of pores trapping the positronium atoms before their decay as well as the pore concentration in all old obsidians are almost the same independently of the place of their formation. Simultaneously the concentration of the pores in the young sample is about five times lower than in the old specimens. Basing on the result it is suggested to extend the study in order to find out if positron annihilation can be used for the investigation of the age of the volcanic accumulations containing obsidian.
\end{abstract}

PACS numbers: $61.43 . F s, 78.70 . B j$

\section{Introduction}

Natural glasses play an important role as a source of information on geological processes [1] and that is why they are intensively studied with various techniques [2]. Among the amorphous materials obsidians are the most frequently found on the Earth surface. They are volcanic glasses which are formed when lava is exposed to the quenching medium. The medium can be ice and water for example as in the case of subglacial eruptions in Iceland [3].

One of the characteristic features of amorphous materials is their porosity. It can be investigated by several techniques among which a family of positron annihilation methods is known as an useful tool. The nondestructive methods use positrons and positronium atoms ( $\mathrm{Ps}$ - the positron-electron bound state) as microscopic probes for the research of matter in different states. Both the 
positron and the positronium atom are preferentially localized at the vacuities of the material under investigation and this reflects on the properties of $\gamma$-rays due to positron-electron annihilation. Thanks to this fact positron annihilation techniques are suitable tools for studying the positron and positronium traps. They can be vacancies and voids in crystals or atomic-scale holes in amorphous materials for example.

In this paper we present the results of our positron annihilation study of the properties of pores in some obsidians. As far as we know the materials have never been investigated with positrons and positronium atoms.

\section{Experimental and results}

The room temperature measurements of lifetime spectra (LS) and one-dimensional angular correlations (AC) of photons coming from the two-photon annihilation of an electron-positron pair were carried out for six dissimilar samples of obsidian originating from Iceland, the Caucasus in Armenia and the Lipari Islands in Italy. The specimens were borrowed from the Museum of Minerals of the Wroclaw University. They were of different age and of similar chemical content characteristic of the rhyolitic obsidian. One specimen (originating from Iceland) was relatively young, formed in 1980 . It has got number 1 in this paper. The five others were much older, found during the 19th century.

LS were collected by using a conventional fast-timing apparatus with $\mathrm{BaF}_{2}$ scintillators, constant fraction differential discriminators (Ortec 583 Model), a time-to-pulse-height converter (Ortec $457 \mathrm{Model}$ ) and a multichannel analyzer data buffer (Ortec 919 Model). The full width at half maximum (FWHM) of the time resolution function of the spectrometer is about $350 \mathrm{ps}$. As the source of positrons the ${ }^{22} \mathrm{Na}$ isotope of the activity of about $4 \times 10^{4} \mathrm{~Bq}$ was used. It was sandwiched between two identically prepared pieces of the sample. The LS measurements were performed in summing-safe, triangular geometry [4]. AC were recorded by means of an automated angular correlation spectrometer with the long-slit geometry and FWIIM of the angular resolution function of about $2 \mathrm{mrad}$. The description of such a spectrometer can be found in [5]: During the $\mathrm{AC}$ measurements specimens were one-sidedly exposed to positrons provided by a $10^{8} \mathrm{~Bq}$ source of ${ }^{22} \mathrm{Na}$.

LS were measured in the time range 0-37 ns making use of 646 channels of the multichannel analyzer. The total number of counts for one LS was about $10^{7}$. The AC measurements were carried out for angles $\theta$ from -34 to $+34 \mathrm{mrad}$ in step of $0.5 \mathrm{mrad}$. The total number of counts for one $\mathrm{AC}$ was $(1.5-2) \times 10^{6}$.

The olitained data were analysed in terms of three-component (3C) models with the computer programs POSITRONFIT [6] and ACPAR [7]. The LS components were exponentials with different mean lifetimes $\left(\tau_{1}, \tau_{2}\right.$ and $\left.\tau_{3}\right)$ and intensities $\left(I_{1}^{\prime}, I_{2}^{\prime}\right.$ and $\left.I_{3}^{\prime}\right)$ whereas the AC components were Gaussians with different FWHM $\left(\Gamma_{1}, \Gamma_{2}\right.$ and $\left.\Gamma_{3}\right)$ and intensities $\left(I_{1}, I_{2}\right.$ and $\left.I_{3}\right)$. The fitted parameters characterizing the mentioned models are presented in Tables I and II. As it is seen corresponding parameters of the models for unlike samples are similar. The only exceptions are the intensity $I_{1}$ of the narrowest Gaussian in the AC for the sample No. 1 - it is the young sample, which is 3 to 4 times smaller than for other samples (old ones) 
TABLE I

The best fit parameters of the three-component model of the $1 \mathrm{D}$ angular correlation of positron annihilation radiation measured for the samples of obsidian; $\chi^{2}$ denotes the variance of the fit.

\begin{tabular}{c|l|c|c|c|c|c|c|c}
\hline \hline No. & \multicolumn{1}{|c|}{ Locality } & $I_{1}[\mathrm{mrad}]$ & $I_{1}[\%]$ & $I_{2}[\mathrm{mrad}]$ & $I_{2}[\%]$ & $I_{3}[\mathrm{mrad}]$ & $I_{3}[\%]$ & $\chi^{2}$ \\
\hline 1 & Iceland & $1.84(48)$ & $\mathbf{0 . 6 ( 1 )}$ & $9.61(3)$ & $90.8(2)$ & $18.7(4)$ & $8.6(1)$ & 1.0 \\
2 & Iceland & $2.24(19)$ & $\mathbf{1 . 8}(1)$ & $9.40(3)$ & $87.5(2)$ & $17.0(3)$ & $10.7(1)$ & 0.9 \\
3 & Caucasus & $2.44(15)$ & $\mathbf{1 . 8}(1)$ & $9.57(2)$ & $89.3(2)$ & $18.2(3)$ & $8.9(1)$ & 1.2 \\
4 & Caucasus & $3.01(22)$ & $2.4(1)$ & $9.69(3)$ & $89.6(2)$ & $19.0(4)$ & $8.0(1)$ & 1.2 \\
5 & Lipari Islands & $2.82(21)$ & $\mathbf{2 . 5}(1)$ & $9.45(4)$ & $88.3(2)$ & $17.9(4)$ & $9.1(2)$ & 1.2 \\
6 & Lipari Islands & $\mathbf{3 . 1 2 ( 2 0 )}$ & $\mathbf{2 . 7 ( 1 )}$ & $9.48(4)$ & $87.9(2)$ & $17.7(4)$ & $9.5(1)$ & 1.2
\end{tabular}

TABLE II

The best fit parameters of the three-component model of the positron lifetime spectrum measured for the samples of obsidian; $\chi^{2}$ denotes the variance of the fit.

\begin{tabular}{c|l|c|c|c|c|c|c|c}
\hline \hline No. & Locality & $\tau_{1}[\mathrm{~ns}]$ & $I_{1}^{\prime}[\%]$ & $\tau_{2}[\mathrm{~ns}]$ & $I_{2}^{\prime}[\%]$ & $\tau_{3}[\mathrm{~ns}]$ & $I_{3}^{\prime}[\%]$ & $\chi^{2}$ \\
\hline 1 & Iceland & $0.403(5)$ & $84.1(4.2)$ & $0.62(4)$ & $15.5(4.0)$ & $1.59(26)$ & $\mathbf{0 . 4 ( 2 )}$ & 1.1 \\
2 & Iceland & $0.404(3)$ & $83.6(2.6)$ & $0.70(5)$ & $14.4(2.0)$ & $1.38(10)$ & $\mathbf{2 . 0}(7)$ & 1.0 \\
3 & Caucasus & $0.406(4)$ & $76.8(3.4)$ & $0.68(3)$ & $21.2(3.1)$ & $1.52(8)$ & $2.0(4)$ & 1.0 \\
4 & Caucasus & $0.417(4)$ & $82.0(2.8)$ & $0.71(5)$ & $16.1(2.2)$ & $1.44(12)$ & $1.9(7)$ & 1.1 \\
5 & Lipari Islands & $0.404(3)$ & $82.0(2.3)$ & $0.70(4)$ & $16.2(1.9)$ & $1.52(10)$ & $1.8(5)$ & 1.1 \\
6 & Lipari Islands & $0.404(5)$ & $80.5(4.1)$ & $0.66(6)$ & $16.7(3.4)$ & $1.33(8)$ & $2.7(5)$ & 1.0
\end{tabular}

as well as the intensity $I_{3}^{\prime}$ of the long-lived component in the LS for the sample No. 1 which is about five times smaller than for other samples. The differences in the central parts of AC for the old and young obsidians are shown in Fig. 1 where the spectra presented are normalized to the same area under the tail parts of them $(|\theta| \geq 5 \mathrm{mrad})$. On the other hand, the differences in the tail parts of LS for the old and young obsidians are demonstrated in Fig. 2 where the spectra presented are normalized to the same height.

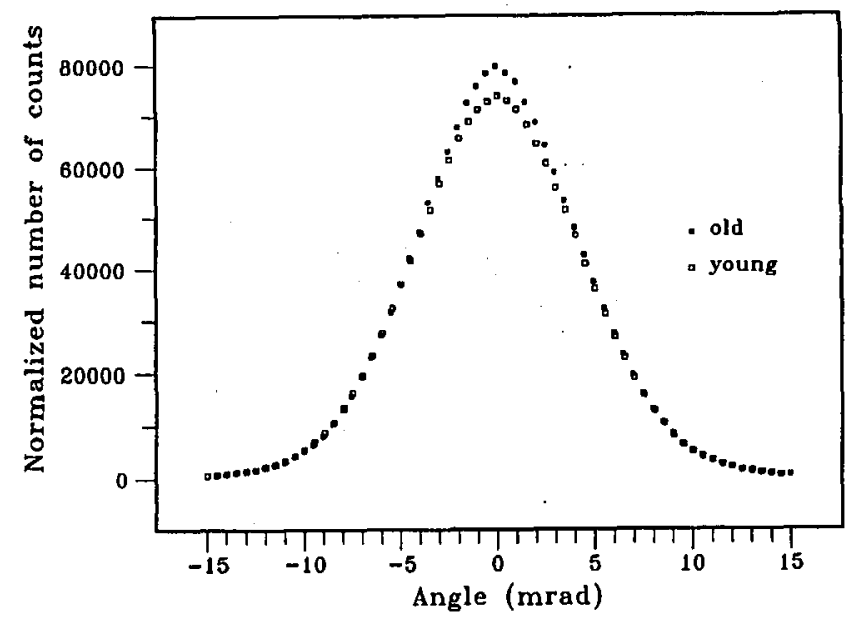

Fig. 1. Angular correlations of positron annihilation radiation normalized to the same area under the tail parts (|angle $\mid \geq 5 \mathrm{mrad}$ ) of them for young (No. 1) and old (No. 2) samples of obsidian from Iceland. 


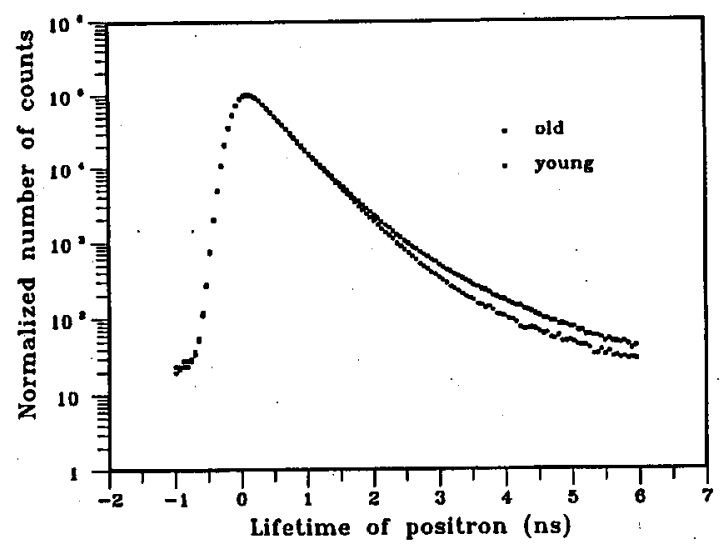

Fig. 2. Positron lifetime spectra normalized to the same height for young (No. 1) and old (No. 2) samples of obsidian from Iceland.

Existing of both the narrow component in $\mathrm{AC}$ (with $\Gamma_{1}$ of about 2-3 mrad) and the long-lived component in LS (with $\tau_{3}$ closed to $1.4 \mathrm{~ns}$ ), observed for all samples studied, speak in favour of the suggestion [8] that the narrow component of AC is associated with the intrinsic annihilation of trapped para-positronium atoms (the total spin $S=0$ ) whereas the long-lived component of LS is conditioned by the pick-off annihilation of positrons forming the trapped ortho-positronium atoms (the total spin $S=1$ ). Taking this into account we used the parameters $\Gamma_{1}$ and $\tau_{3}$ for determining characteristics of pores trapping the positronium atoms before their decay. It was done accepting the commonly used simple models of a pore $[9,10]$ according to which the pore constitutes for positronium a square potential well of spherical symmetry and assuming that positronium trapped in the well occupies the ground state before its decay. The proper computations were performed for the well without electrons inside, having the potential barrier of a finite height $[9,11]$ (the model I) and for the well with a layer of electron cloud inside, located at the surface of the well barrier of an infinite height $[10,12]$ (the model II). As a result the depth $U$. (the model I) and the radius $R$ of the wells in the studied samples were determined. In the calculations we used the relationships given in the literature. In the first case they were taken from the publication of Bartenev et al. [11]:

$$
\begin{aligned}
& R_{U}=x \frac{2 h}{m_{0} c \Gamma_{1}} \\
& U=\frac{m_{0} c^{2} \Gamma_{1}^{2}}{16 \sin ^{2}(x)},
\end{aligned}
$$

where $x$ is a solution of the following equation:

$$
\frac{\tau_{0}}{\tau_{3}}=\frac{\sin ^{3}(x)}{\sin (x)+x\left[1-\sin ^{2}(x)\right]^{1 / 2}}
$$

under the condition $\operatorname{ctg} x<0, h$ is the Planck constant divided by $2 \pi, m_{0}$ denotes the electron mass, $c$ stands for the velocity of light and $\tau_{0}=350 \mathrm{ps}$. In the second 
case the used formulas were taken from the papers by Eldrup et al. [12] and Briscoe et al. [13]:

$$
\begin{aligned}
& \tau_{3}=\frac{1}{2}\left[1-\frac{R_{\infty}^{\tau}-\Delta R}{R_{\infty}^{\tau}}+\frac{1}{2 \pi} \sin \left(2 \pi \frac{R_{\infty}^{\tau}-\Delta R}{R_{\infty}^{\tau}}\right)\right]^{-1}, \\
& R_{\infty}^{\Gamma}=2.148 \frac{2 h}{m_{0} c \Gamma_{1}}
\end{aligned}
$$

where $\Delta R=166 \mathrm{pm}$ is an empirical parameter describing the thickness of a layer of electron cloud located inside the well at the surface of its barrier and $\tau_{3}$ is expressed in the units of ns. As it is seen from the above equations, in the second case the radius $R$ of the positronium traps can be determined independently from $\mathrm{AC}\left(R_{\infty}^{\Gamma}\right)$ and LS $\left(R_{\infty}^{\tau}\right)$ data whereas in the first case determining of $R\left(R_{U}\right)$ requires using both $A C$ and LS parameters simultaneously. The obtained results are collected in Table III.

TABLE III

Parameters of positronium traps in the samples of obsidian.

\begin{tabular}{c|l|l|l|l|l}
\hline \hline No. & \multicolumn{1}{|c|}{ Locality } & $R_{U}[\mathrm{~nm}]$ & $U[\mathrm{eV}]$ & $R_{\infty}^{\tau}[\mathrm{nm}]$ & $R_{\infty}^{\Gamma}[\mathrm{nm}]$ \\
\hline 1 & Iceland & $0.94(25)$ & $0.18(9)$ & $0.41(3)$ & $0.90(24)$ \\
2 & Iceland & $0.76(7)$ & $0.24(4)$ & $0.39(2)$ & $0.74(6)$ \\
3 & Caucasus & $0.71(5)$ & $0.30(4)$ & $0.40(1)$ & $0.68(4)$ \\
4 & Caucasus & $0.57(4)$ & $0.45(7)$ & $0.39(2)$ & $0.55(4)$ \\
5 & Lipari Islands & $0.61(5)$ & $0.41(6)$ & $0.40(2)$ & $0.59(5)$ \\
6 & Lipari Islands & $0.54(4)$ & $0.47(6)$ & $0.38(1)$ & $0.53(4)$
\end{tabular}

On the basis of the data we estimated the mean radius $r$ of the free volumes in the obsidian samples using the following simple relations:

$$
r_{U}=R_{U}, \quad r_{\infty}^{\Gamma}=R_{\infty}^{\Gamma}-\Delta R, \quad r_{\infty}^{\tau}=R_{\infty}^{\tau}-\Delta R .
$$

The parameters are presented in Table IV.

TABLE IV

Parameters of free volumes in the samples of obsidian.

\begin{tabular}{c|l|l|l|l|l|c}
\hline \hline No. & \multicolumn{1}{|c|}{ Locality } & $r_{U}[\mathrm{~nm}]$ & $r_{\infty}^{\Gamma}[\mathrm{nm}]$ & $r_{\infty}^{\tau}[\mathrm{nm}]$ & $r_{\infty}[\mathrm{nm}]$ & $\Delta R[\mathrm{~nm}]$ \\
\hline 1 & Iceland & $0.94(25)$ & $0.74(24)$ & $0.24(3)$ & $0.54(25)$ & 0.37 \\
2 & Iceland & $0.76(7)$ & $0.58(6)$ & $0.22(2)$ & $0.42(7)$ & 0.32 \\
3 & Caucasus & $0.71(5)$ & $0.52(4)$ & $0.24(1)$ & $0.40(5)$ & 0.28 \\
4 & Caucasus & $0.57(4)$ & $0.39(4)$ & $0.23(2)$ & $0.32(4)$ & 0.23 \\
5 & Lipari Islands & $0.61(5)$ & $0.42(5)$ & $0.24(2)$ & $0.35(5)$ & 0.24 \\
6 & Lipari Islands & $0.54(4)$ & $0.37(4)$ & $0.21(1)$ & $0.30\left(4^{\prime} ;\right.$ & 0.23
\end{tabular}




\section{Conclusions}

1. There are empty pores inside the studied samples of obsidian that trap positronium atoms before their decay.

2 . The values of the mean radius $r$ of the free volumes "seen" by the positronium atoms depend on the method of their estimation. The $r$ values resulting from the commonly accepted interpretation of the positron annihilation data range from 0.5 to $0.9 \mathrm{~nm}$ and from 0.2 to $0.7 \mathrm{~nm}$ in the case of using the positronium trap model I and II respectively.

3 . The values of $r_{\infty}^{\tau}$ are essentially smaller than the ones of $r_{\infty}^{\Gamma}$. This may suggest that the accepted three-Gaussian model of $A C$ is not valid for obsidians and the main component of AC should be described by another function (non-Gaussian) with greater FWHM. On the other hand, the formulas (4) and (5) for positronium in solids were derived using the positronium wave function in vacuum and this simplification can also be responsible for observed differences between $r_{\infty}^{\tau}$ and $r_{\infty}^{\Gamma}$ values. Finally, the mentioned differences can be explained assuming that the thickness $\Delta R$ of the electron layer inside the model pore (II) is different for dissimilar materials (pores) and for obsidians it is greater than $0.17 \mathrm{~nm}$, accepted in our computation following [12]. Similar discrepancy between the $r_{\infty}^{\tau}$ and $r_{\infty}^{\Gamma}$ values were observed by us for some naturally occurring silica materials [14] and amber [15] whereas Jean [16] obtained an excellent agreement between the values mentioned for an epoxy polymer studied in various temperatures. Assuming that $r_{\infty}^{\Gamma}=r_{\infty}^{\tau}=r_{\infty}$ one can find that for the obsidian samples $\Delta R$ changes from $0.23 \mathrm{~nm}$ to $0.37 \mathrm{~nm}$ and $r_{\infty}$ varies from 0.3 to $0.5 \mathrm{~nm}$. The obtained values of $r_{\infty}$ and $\Delta R$ for studied specimens are shown in Table IV.

4. The concentration of the pores detected by positronium atoms (related to the $I_{1}$ and $I_{3}^{\prime}$ parameters) in the young sample from Iceland is several times smaller than in the old one. The result is interesting as it suggests that positron annihilation technique may be useful in the age estimation of the volcanic accumulations containing obsidian. It seems however that drawing the final conclusions on the question requires further experimental data of the kind presented in this paper.

TABLE V

The best fit parameters of the two-component model of the positron lifetime spectrum measured for the samples of obsidian.

\begin{tabular}{c|l|c|c|c|c|c}
\hline \hline No. & \multicolumn{1}{|c|}{ Locality } & $\tau_{1}[\mathrm{~ns}]$ & $I_{1}[\%]$ & $\tau_{2}[\mathrm{~ns}]$ & $I_{2}[\%]$ & $\chi^{2}$ \\
\hline 1 & Iceland & $0.415(1)$ & $93.4(4)$ & $0.81(1)$ & $6.6(4)$ & 1.2 \\
2 & Iceland & $0.418(1)$ & $91.5(2)$ & $0.99(1)$ & $8.5(2)$ & 1.2 \\
3 & Caucasus & $0.431(1)$ & $91.5(2)$ & $1.02(1)$ & $8.5(2)$ & 1.5 \\
4 & Caucasus & $0.433(1)$ & $91.0(2)$ & $1.00(1)$ & $9.0(2)$ & 1.3 \\
5 & Lipari Islands & $0.421(1)$ & $91.4(2)$ & $1.02(1)$ & $8.6(2)$ & 1.5 \\
6 & Lipari Islands & $0.421(1)$ & $91.5(2)$ & $1.01(1)$ & $8.5(2)$ & 1.2
\end{tabular}


5. As a final point we would like to return to the LS for the samples studied. It seems that an analysis of the spectra in the terms of the two-component (2C) model can be fruitful from the mineralogy point of view. The results of such analysis (Table V) suggest that the $\tau_{1}$ and $\tau_{2}$ parameters of the $2 \mathrm{C}$ model may be related to the place of formation (chemical content) and the age of obsidian, respectively. The hypothesis will be checked in the nearest future. At the same time one should note that the $2 \mathrm{C}$ model of LS is not proper for obsidians in the light of the $\chi^{2}$ value for the fit. The $\chi^{2}$ value increases with statistics - see Fig. 3 .

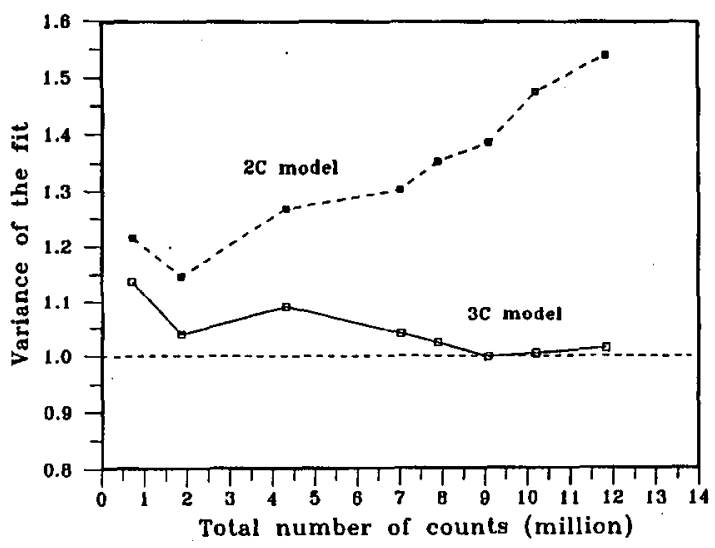

Fig. 3. The variance $\chi^{2}$ of the fit for two and three component models of the positron lifetime spectrum measured for the sample No. 3 (obsidian from the Caucasus in Armenia) as a function of the statistics of the spectrum.

\section{Acknowledgments}

This work has been sponsored by the State Committee for Scientific Research (Republic of Poland) in the frame of the project No. 604589101.

\section{References}

[1] Prirodnyje stekla - indikatory geologicheskikh processov, Eds. O.A. Bogatikov, A.M. Borsuk, Nauka, Moskva 1987.

[2] Proc. Second Int. Conf. on Natural Glasses, Prague 1987, Ed. J. Konta, Charles University, Praha 1988.

[3] H. Furnes, I.B. Fridleifsson, F.B. Atkins, J. Volcanology Geotherm. Res. 8, 95 (1980).

[4] T. Goworek, W. Górniak, J. Wawryszczuk, Acla Phys. Pol. A 83, 307 (1993).

[5] B. Rozenfeld, A. Baranowski, K. Jerie, Nukleonika 19, 817 (1974).

[6] P. Kirkegaard, N.J. Pedersen, M. Eldrup, PATFIT-88: A Data-Processing System for Positron Annihilation Spectra on Mainframe and Personal Computers, Tech. Rep. Ris $\varnothing-M-2740$, Ris $\varnothing$ National Laboratory, Roskilde 1989. 
[7] A. Baranowski, Program $A C P A R$, in a software of the Computer Library of the Department of Applied Nuclear Physics at the Wroclaw University.

[8] S.J. Wang, Y.C. Jean, in: Positron and Positronium Chemistry, Eds. D.M. Schrader, Y.C. Jean, Elsevier, Amsterdam 1988, p. 266.

[9] W. Brandt, S. Berko, W.W. Walker, Phys. Rev. 120, 1289 (1960).

[10] S.J. Tao, J. Chem. Phys. 56, 5499 (1972).

[11] G.M. Bartenev, A.D. Cyganov, A.Z. Varisov, Izv. Vyssh. Uchebn. Zaved. Fiz. 5, 59 (1971).

[12] M. Eldrup, D. Lightbody, J.N. Sherwood, Chem. Phys. 63, 51 (1981).

[13] C.V. Briscoe, S.I. Choi, A.T. Stewart, Phys. Rev. Lett. 20, 493 (1968).

[14] J. Chojcan, M. Sachanbiniski, Acta Phys. Pol. A 83, 267 (1993).

[15] J. Chojcan, M. Sachanbinski, in: Proc. XXVIII Zakopane School of Physics on Condensed Matter Studies by Nuclear Methods, Eds. E.A. Görlich, K. Tomala, Jagiellonian Univ. \& Inst. of Nucl. Phys., Cracow 1993, p. 296.

[16] Y.C. Jean, Nucl. Instrum. Methods Phys. Res. B 56/57, 615 (1991). 